\title{
Improved Parametric Imaging of Scatterer Size Estimates Using Angular Compounding
}

\author{
Anthony L. Gerig, Student Member, IEEE, Tomy Varghese, Senior Member, IEEE, \\ and James A. Zagzebski, Associate Member, IEEE
}

\begin{abstract}
The feasibility of estimating and imaging scatterer size using backscattered ultrasound signals and spectral analysis techniques was demonstrated previously. In many cases, size estimation, although computationally intensive, has proven to be useful for monitoring, diagnosing, and studying disease. However, a difficulty that is encountered in imaging scatterer size is the large estimator variance caused by statistical fluctuations in echo signals from random media. This paper presents an approach for reducing these statistical uncertainties. Multiple scatterer size estimates are generated for each image pixel using data acquired from several different directions. These estimates are subsequently compounded to yield a single estimate that has a reduced variance. In this feasibility study, compounding was achieved by translating a sectored-array transducer in a direction parallel to the acquired image plane. Angular compounding improved the signal-to-noise ratio (SNR) in scatterer size images. The improvement is proportional to the square root of the effective number of statistically independent views available for each image pixel.
\end{abstract}

\section{INTRODUCTION}

$\mathrm{U}$ LTRASOUND is widely regarded as a safe, cost effective, and versatile medical imaging modality. It is used in numerous applications, including imaging the abdominal organs, fetus, and breast. It also is used for guiding and monitoring interventional procedures, such as biopsies and minimally invasive ablation therapy of tumors. The modality continues to grow, with new scanning machines and applications emerging every year.

Despite its success as an imaging modality and its effectiveness in many types of clinical procedures, the echo signal information used in current scanners represents only a fraction of the information content contained in these signals. In gray scale imaging, the most widely used mode, only the amplitudes of the echo signal are detected and displayed. Phase and echo frequency information are ignored, but information in these signals can lead to new ultrasound images with potentially greater information content than that of current methods.

Research in ultrasonic "tissue characterization" supports the assertion that ultrasound signals contain considerably more information about the tissue being imaged

Manuscript received June 23, 2003; accepted January 12, 2004. This work was supported in part by start-up grant funds from the Department of Medical Physics, Medical School and Graduate School at the University of Wisconsin-Madison and by NIH grants R01 CA39224, R21 EB002722, and T32CA09206.

The authors are with The University of Wisconsin-Madison, Madison, WI 53706 (e-mail: algerig@students.wisc.edu). than what is currently used [1]-[10]. Ultrasound waves interact with tissue such that information in the backscattered radio frequency (RF) signal can be used to identify and monitor tissue microstructure and disease properties [1]-[5]. This difference in structure is believed to manifest itself in the frequency dependence of scattering of ultrasound waves [1]-[10]. Different tissues exhibit different ultrasonic wave propagation phenomena, and these phenomena can be characterized by an attenuation parameter and descriptors of the "effective scatterers" such as shape, size, and number density.

Many investigators have measured the frequency dependence of scattering to estimate parameters related to scatterer size [4], [5], [9]-[11]. In addition, other parameters such as the scatterer spacing [8], [12], [13], and scatterer number density [1], [14]-[16] have been estimated, with varying degrees of success. For example, in liver tissue, scattering from two types of structures has been observed, in which the lobular structure of the liver and portal triads contribute to a resolvable or quasiperiodic component on the order of $1 \mathrm{~mm}$. Rayleigh-like scattering is generated from the numerous and randomly distributed hepatocytes [12], [17]. Insana et al. [4], produced scatterer size images of kidneys; their results agree well with glomerular diameters measured from pathology specimens of the scanned kidney samples. Using their parametric imaging techniques, they were able to rapidly assess changes in the average glomerular diameter and the average arteriolar cross-sectional diameter in ischemic dog kidney [18]. Sommer et al. [19] demonstrated the utility of viewing liver tumors using multiple narrowband frequency images. The B-mode contrast between tumors and background liver tissue in their subjects varied with ultrasound frequency and with tumor type. Furthermore, this frequency-dependent contrast depended on the sizes of internal structures of tumors and normal liver viewed under the microscope.

Much of the early work in scatterer size estimation involved the use of single-element transducers, although in subsequent years work has progressed to accommodate array transducers and clinical systems through the development of novel techniques to account for system dependencies [20]-[22]. Our own work in scatterer size imaging demonstrates not only that accurate parametric images of effective size can be obtained, but that the statistical errors are also understood [23].

The purpose of the work described in this paper was to assess feasibility of reducing statistical errors in scatterer size images by compound data acquisition. Com- 
pound imaging is used on some clinical scanners to reduce the speckle noise on B-mode images. These machines steer beams from linear or curvilinear arrays in different directions, so that echo signals from each location imaged are acquired from various directions then combined. We believe that this compound acquisition approach, which until now has not been implemented in quantitative ultrasound imaging, is promising for parametric imaging on clinical instruments.

\section{THEORY}

The method used for producing scatterer size images is described in detail elsewhere [23], so it will only be summarized briefly here. Spatially dependent computations of the scatterer size parameter are done by performing a modified least squares fit between the measured backscatter coefficient from a tissue segment, $B S C_{s}(\omega)$ and a scatterer size $(a)$ dependent, theoretical backscatter coefficient, $B S C_{t}(\omega, a)[4]$. The approach searches for a scatterer size that provides the best fit to the measured backscatter coefficient data over the frequency bandwidth of the measurement. Thus, the scatterer size, $\hat{a}$ is estimated using:

$$
\hat{a}=\arg \min \frac{1}{n} \sum_{\omega_{\min }}^{\omega_{\max }}[\psi(\omega, a)-\bar{\psi}(a)]^{2}
$$

where:

$$
\psi(\omega, a)=10 \ln \left[B S C_{s}(\omega)\right]-10 \ln \left[B S C_{t}(\omega, a)\right]
$$

is a cost function, $\omega$ is the angular frequency, $\omega_{\min }$ and $\omega_{\max }$ represent the lower and upper bound of the frequency bandwidth, and $\bar{\psi}(a)$ is the mean value of $\psi(\omega, a)$ over the frequency bandwidth. Measurements of backscatter coefficients are obtained using a reference phantom to account for system effects, including beam properties, transmit pulse amplitude, receiver gain, and other signal processing [22], [24]. The general approach assumes no significant refraction or phase aberration.

Size estimates generated using this method are inherently noisy due to the large variance of spectral estimates. The variance, assuming a Gaussian spatial autocorrelation function, has been calculated [23], [25] to be:

$$
\operatorname{var}(\hat{a}) \approx \frac{c^{4} d_{1}^{4}}{16^{2} a^{2}}\left(\frac{N_{s}+N_{r}}{N_{s} N_{r}}\right) \frac{1}{\sum_{\omega_{\min }}^{\omega_{\max }}\left(\omega^{2}-\overline{\omega^{2}}\right)^{2}}
$$

where $c$ is the speed of sound, and the constant $d_{1}$ [25] relates the scatterer diameter to the effective length of the correlation function used to obtain the theoretical backscatter coefficient. $N_{s}$ and $N_{r}$ are the number of independent sample and reference power spectra averaged in estimating the backscatter. The summation is limited to frequencies for which the associated backscatter estimates are statistically uncorrelated with one another. The frequency interval between uncorrelated estimates is a function of data window type and is inversely proportional to window length. (Details are reported in Gerig et al. [23].)

Although estimate precision can be increased by using higher frequencies, larger bandwidths, and longer data windows, the improvement that these options afford is generally limited by other factors and by the desire to limit image pixel size. Another possibility for reducing size estimate variance and improving the associated signal-to-noise ratio (SNR) is to compound either size or backscatter estimates (expected improvement identical) taken from different spatial locations that contain scatterers of the same type. For example, the optimal $\sqrt{N}$ improvement in image SNR, where $\mathrm{N}$ is the number of averaged estimates for each pixel, can be achieved for parametric images of scatterer size by compounding parallel images spaced along the direction perpendicular to the image/scan plane, i.e., the elevational direction. However, this method is effective only when the imaged object possesses symmetry in the elevational direction, such that the compounded parametric images possess identical structure but different noise patterns.

With angular compounding, partially correlated echo data are collected from the same spatial location but from different angles of incidence. The same scatterers contribute to the data for each angle of incidence, but signals are partially decorrelated due to the change in relative scatterer position with respect to the transducer aperture upon rotation. (Note that this explanation assumes significant overlap of the gated fields with rotation.) Imaging performance thus can be improved with this type of spatial compounding, despite the absence of object symmetry.

\section{MAterials AND Method}

A mechanically translated sector transducer was used to acquire compound data for this study. The RF echo signal data were obtained using an Aloka SSD 2000 (Aloka Inc. Tokyo, Japan) real-time scanner using a $3.5 \mathrm{MHz}$ phasedarray transducer with an approximately $70 \%$ bandwidth. Each RF frame consisted of 121 A-lines (due to triggering delay, the first 6 lines of every frame were not recorded) evenly spaced over a $90^{\circ}$ sector, resulting in an angular increment of 0.75 degrees. The ultrasound RF signals were digitized using a 12-bit data acquisition board (Gage Inc., Montreal, Canada) at a sampling rate of $50 \mathrm{MHz}$. To achieve the effects of compound data acquisition, the phased-array transducer was linearly translated over the sample using a precision linear stage. Probe translation was in the lateral direction in the image plane, so that most locations in the sample were scanned from multiple angles, as illustrated in Fig. 1. Data sets were acquired and stored for 64 transducer locations separated laterally by increments of $0.5 \mathrm{~mm}$.

Echo data were obtained from a specially constructed agar phantom [Fig. 2(a)] that contained cylindrical inclu- 

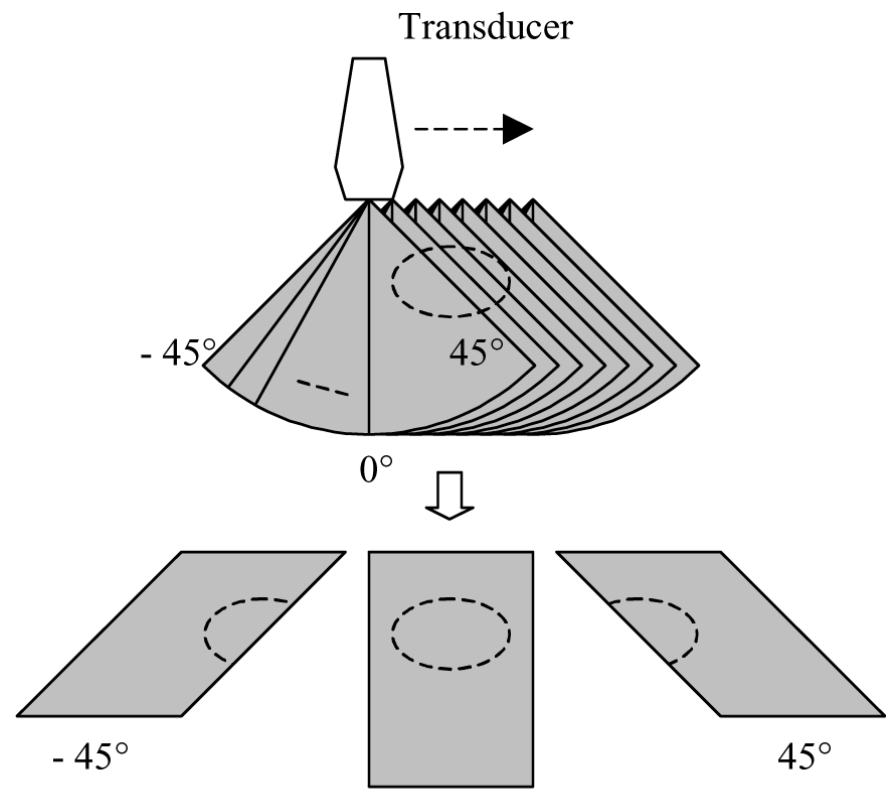

$0^{\circ}$

Fig. 1. RF data sets in sector format (top) were acquired at each lateral position. These scans were rearranged into linear scans (bottom) by grouping A-lines at the same angle. Each frame of the linear scan presents an RF data set from a different angle.

sions, each having a different scatterer size. Scattering objects in each inclusion were spherical polystyrene beads, whose size distributions are shown in Table I. When viewed on B-mode images, a gray scale contrast exists between the background and the inclusions containing the smaller scatterers [Fig. 2(b)]; however, at $3.5 \mathrm{MHz}$, the cylinders containing larger scatterers are nearly isoechoic relative to the background. The background and inclusions have a speed of sound of $1540 \mathrm{~m} / \mathrm{s}$ and an attenuation coefficient of approximately $0.43 \mathrm{~dB} / \mathrm{cm}-\mathrm{MHz}$.

Multiple ultrasound RF data sets were acquired as described previously. The RF data then were reformatted into sets that represented planes with scan angles of $0^{\circ}$, $\pm 0.75^{\circ}, \pm 1.5^{\circ}$, etc. to an angular range of $\pm 45^{\circ}$. Multiple planes of data also were acquired from a reference phantom, using identical system settings, for backscatter estimation. Backscatter coefficients were generated by comparing echo spectra from relevant locations in the test sample with reference spectra from the corresponding locations in the reference phantom. The 5-mm axial data segments were used to estimate spectra, and point attenuation corrections were made [24]. Size estimates were generated for each angular plane in increments of $2.5 \mathrm{~mm}$ axially, following the method outlined in (1). Although image resolution could have been improved by using shorter data segments, the trade-off would have been an increase in size estimate standard deviation. A Gaussian spatial autocorrelation model was used to estimate scatterer size, despite the fact that it is not completely accurate for polystyrene beads, and that some of the scatterer sizes were larger than recommended for accurate estimation results at $3.5 \mathrm{MHz}$ [4]. Therefore, size estimates were expected to be biased.
TABLE I

Polystyrene Bead Size Distribution Information for the Scatterer-Size Phantom.

\begin{tabular}{lcc}
\hline & $\begin{array}{c}\text { Mean diameter } \\
\text { (microns) }\end{array}$ & $\begin{array}{c}\text { Standard deviation } \\
\text { (microns) }\end{array}$ \\
\hline Background & 200 & 14 \\
Cylinder 1 & 335 & 12 \\
Cylinder 2 & 232 & 8 \\
Cylinder 3 & 99 & 14 \\
Cylinder 4 & 51 & 3 \\
\hline
\end{tabular}

However, because this work is concerned primarily with improvements in estimate noise (standard deviation), the implications were minimal.

Each estimate then was registered to its correct location in space using a transformation/interpolation algorithm. The interpolation process itself may have introduced errors that scale with the distance between size estimates on the image grid. In particular, the correlation between size estimates at different angles may have been artificially reduced, leading to a measurable underestimation of the standard deviation for compounded image data. The 115 individual scatterer size images, each corresponding to a different beam angle in the phased-array data acquisition, were generated (see Fig. 1). A compound image could be produced using any combination of these angled plane data sets by averaging over the estimates available for each spatial location.

\section{Results}

Improvements in the scatterer size images obtained using various angular compounding schemes are described in this section. Fig. 3(a) is a B-mode image of the phantom obtained by envelope detection only of the RF data from the central, vertical line of the sector transducer for each of the 64 transducer locations used in data acquisition; Fig. 3(b) is a scatterer-size image produced using the same data. Half of each cylinder is included in the field of view, i.e., halves of cylinders 1 and 3 appear on the left at depths of $6.5 \mathrm{~cm}$ and $3.5 \mathrm{~cm}$, respectively; and halves of cylinders 2 and 4 appear on the right. Notice that these images do not include spatial compounding. Although the cylinders are visible in the scatterer-size image, their contrast with respect to the background is clearly marred by high-size estimate noise. Cylinder 3, in particular, which is characterized by a size estimate standard deviation much higher than that for cylinder 2 and a bead size much closer to the background than cylinder 4 , is essentially indistinguishable from the background.

Fig. 4 presents scatterer-size images that incorporate spatial compounding. The field of view is larger than that of Fig. 3 because of the use of angled beam data. Visual inspection suggests that the estimation noise is dramatically reduced in the size images of Fig. 4 compared to that of Fig. 3(b). In particular, the top two cylindrical inclu- 


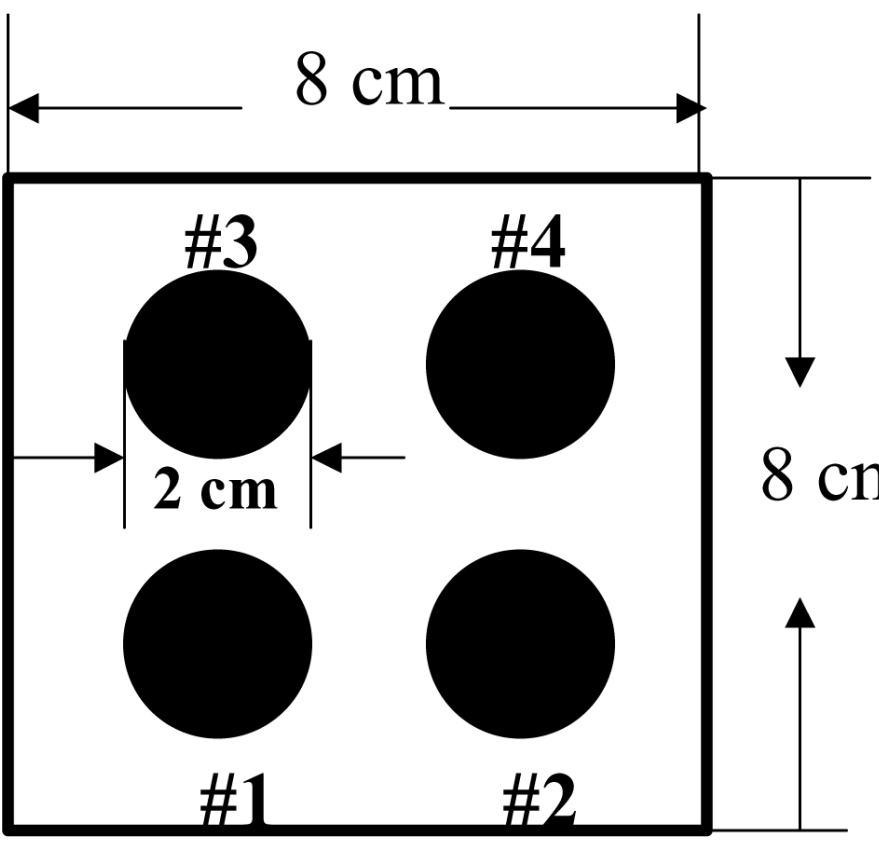

(a)

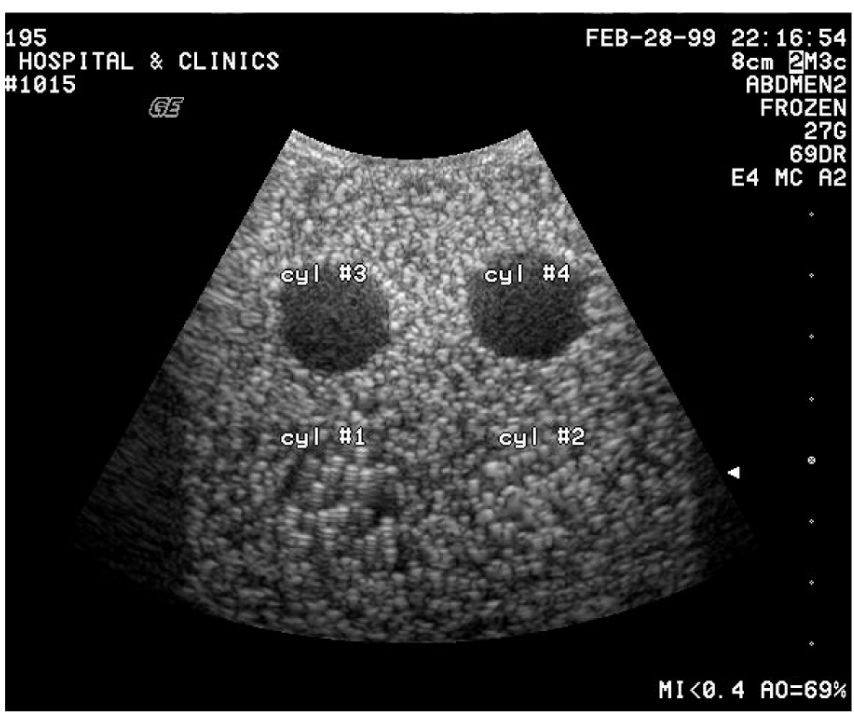

(b)

Fig. 2. A schematic diagram (a) and B-mode image (b) of the scatterer-size phantom.

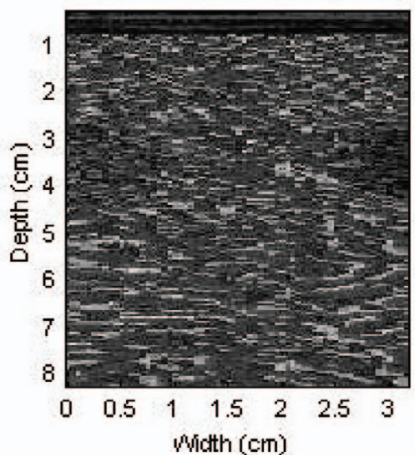

(a)

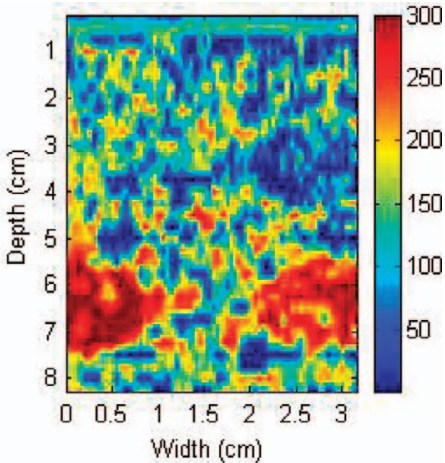

(b)
Fig. 3. This figure illustrates the (a) ultrasound B-mode image and the (b) individual parametric image of scatterer size (diameter in microns) obtained at an angle of 0 degrees.

sions are more clearly distinguished from the background in the compounded images. The boundaries of the bottom two cylinders are sharper, and estimates are more homogeneous both for the cylinders and the background. Fig. 4(a) was obtained using an angular increment of $\pm 0.75^{\circ}$ between beam lines used to form the compounded image. In a similar manner, Figs. 4(b) and (c) use angular increments of $\pm 3^{\circ}$ and $\pm 7.5^{\circ}$, respectively, obtained by skipping beam lines and selecting only every fourth [Fig. 4(b)] or every tenth [Fig. 4(c)] angular plane from the 115 available.

Fig. 5 displays the number of size estimates available at each spatial location for the images in Fig. 4. As many as 115 estimates, not all of which were uncorrelated, were available. It is expected that the noise in the compounded images would be more pronounced in regions in which fewer estimates were available for compounding, which for the present scan format is near the lateral margins and at the larger depths.

The mean and standard deviation of the scatterer-size estimates generated for each cylinder are shown in Fig. 6, plotted as a function of beam angle with respect to vertical. Values were obtained by forming regions of interest that covered approximately half of the projected area of each cylinder (delineated by what appears of each cylinder in Fig. 3) within the pertinent angled planes. Note that the mean scatterer size remains steady over the range of plotted plane angles for the four different scatterer sizes. As expected, the estimates are biased and appear to become worse with increasing size. However, the standard deviation reduces with an increase in the angle, particularly for the cylinders with the smaller scatterer sizes (cylinders 3 and 4), but less so for the cylinders containing the larger scatterer sizes (cylinders 1 and 2). The source of this decreased standard deviation is unclear, although it appears to stem from an observed yet ill-characterized compression of the central RF line signals by the imaging system.

Results of an analysis of scatterer-size statistics for increasing degrees of angular compounding are shown in Figs. 7 and 8. The analysis was done for cylinders 1 and 4 , both clearly demarcated in the scatterer-size images of Fig. 4. In both cases, the standard deviation of compounded size estimates over the projected area of the inclusion were computed as additional angled planes were compounded in the image. Angled planes were added out to a maximum angle of $15^{\circ}$. Four different angular increments between beams were considered. The $0.75^{\circ}$ angular increment used every beam angle acquired (21 over the $15^{\circ}$ range considered here); the $1.5^{\circ}$ increment used every other beam angle, etc. The total number of beam angles available decreases with an increase in the angu- 


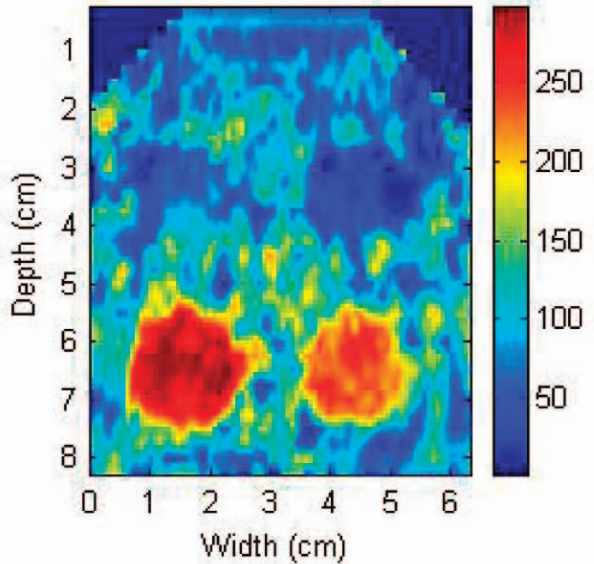

(a)

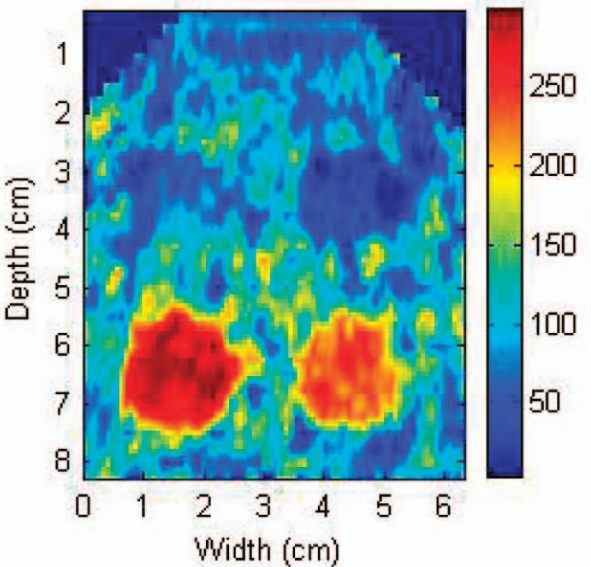

(b)

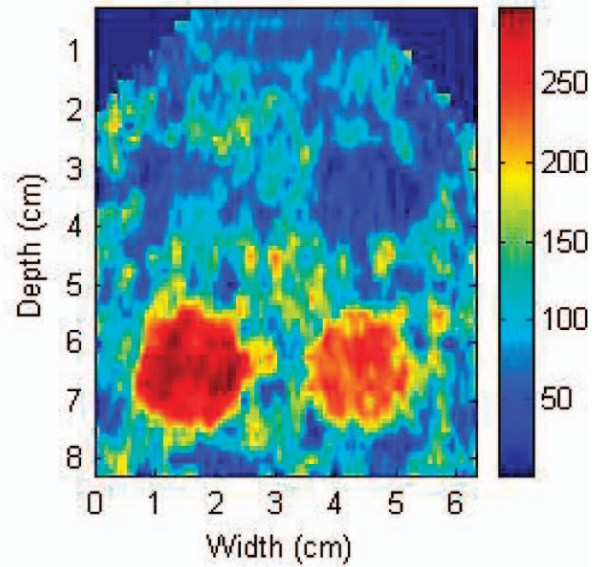

(c)

Fig. 4. Compounded images of scatterer size (diameter in microns) using data from every (a) 0.75 degrees, (b) 3 degrees, and (c) 7.5 degrees.

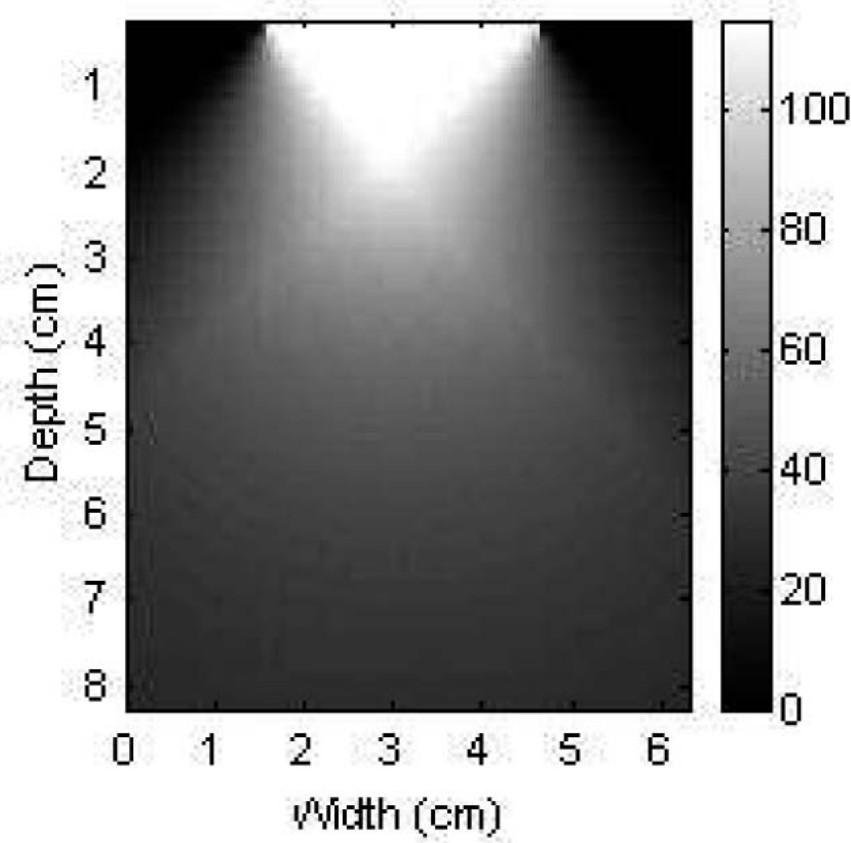

Fig. 5. The number of angular size estimates available at each location of the imaging field.

lar increment. However, the most notable improvements in standard deviation are attained with angular compounding when less correlated data sets, corresponding to larger angular increments, are averaged. Thus, for a given maximum acquisition angle, a trade-off exists between the angular increment and the achievable standard deviation.

The results for cylinder 1 are shown in Fig. 7. The greater standard deviation for the angular increment of $7.5^{\circ}$ is related to a smaller number of planes used in compounding, with only two planes available at a maximum angle of $7.5^{\circ}$, and three at a maximum angle of $15^{\circ}$. However, note the significant reduction in the standard deviation of the estimates, from $24 \mu$ to about $17 \mu$ for a maximum angle of $6^{\circ}$, and to approximately $14 \mu$ at $15^{\circ}$.
The size estimates for the smaller beads of cylinder 4 are inherently noisier than those for the larger beads of cylinder 1, as expected based upon the form of (2). The standard deviation reduces from about $58 \mu$ without compounding to about $30 \mu$ for a maximum angle of $3^{\circ}$, to $24 \mu$ at $15^{\circ}$. The trade-off between the angular increment and estimate variance is clearly observed in Fig. 8.

The standard deviation of the scatterer-size estimates versus the number of estimates averaged is shown in Fig. 9 for cylinders 1 and 4. Also superimposed on the graphs is the optimal $1 / \sqrt{N}$ curve, where $\mathrm{N}$ represents the number of averaged estimates. The standard deviation should follow this curve if estimates are uncorrelated. This optimal behavior is seen for angular increments of $3.5^{\circ}$ and higher, particularly for cylinder 4 , which is closer to the transducer than cylinder 1 . Nevertheless, in either case the larger angular increments provide a faster reduction in the standard deviation with increasing number of estimates.

\section{Discussion and Conclusions}

Traditional parametric images of scatterer size utilize RF-data segments obtained along a single angle of incidence. To produce compounded images of scatterer size, multiple, uncorrelated, or partially correlated RF-data segments can be acquired from the same tissue region at different angles of incidence. In this paper, we translated a phased-array transducer using a precision linear stage to acquire RF data from locations in a phantom at different incident angles. Resultant scatterer-size images exhibit higher SNR with greater degrees of angular compounding.

Angular compounding was achieved in this paper by laterally translating a phased-array transducer to interrogate each region in the scan plane at several angles. A more efficient approach for clinical ultrasound systems will be to steer the ultrasound beam electronically to the same region from different active areas of the transducer face, such that multiple beam lines pass over the same region. This type of beam steering and compound imaging is avail- 


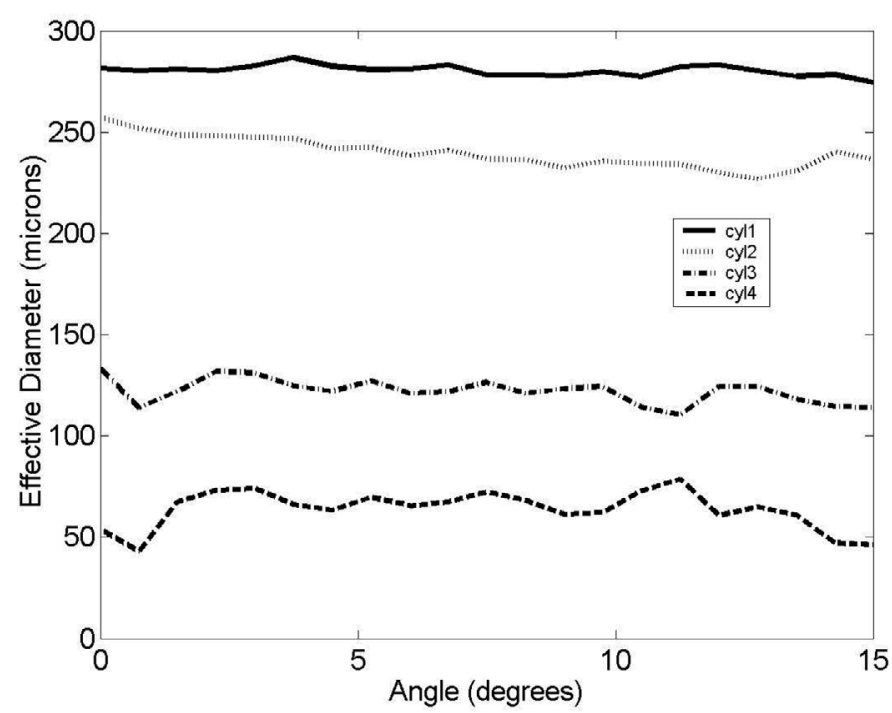

(a)

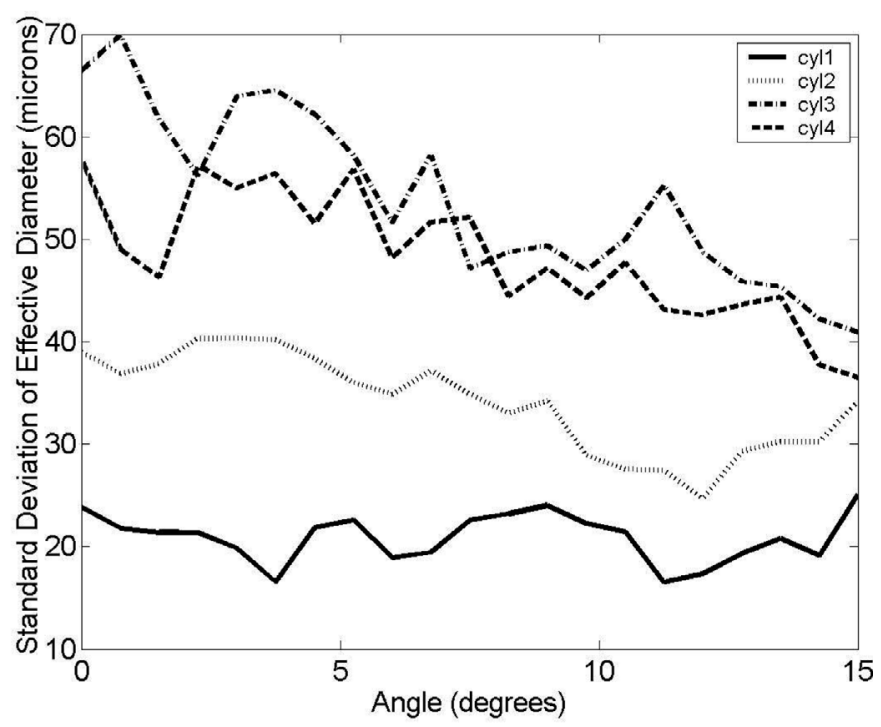

(b)

Fig. 6. Plots of size estimate (a) mean and (b) standard deviation as a function of acquisition angle for the four cylinders contained in the scatterer-size phantom.

able for gray scale imaging, but currently RF data are not widely available for such modes of operation.

In the implementation of angular compounding, a trade-off was seen between the angular increment used and the standard deviation of size estimates. This is caused by correlations among the data for small, angular increments. If the correlation coefficient between size estimates associated with an individual spatial location is known as a function of the angular separation between incident beams, it becomes possible not only to determine theoretical standard deviation values for Figs. 7, 8, and 9, but also to optimize the trade-off between angular increment and the noise in size estimates. In general, the correlation between estimates acquired from different beam angles can be a complicated function of scatterer position and system parameters. Rather than calculate it for our system, it was

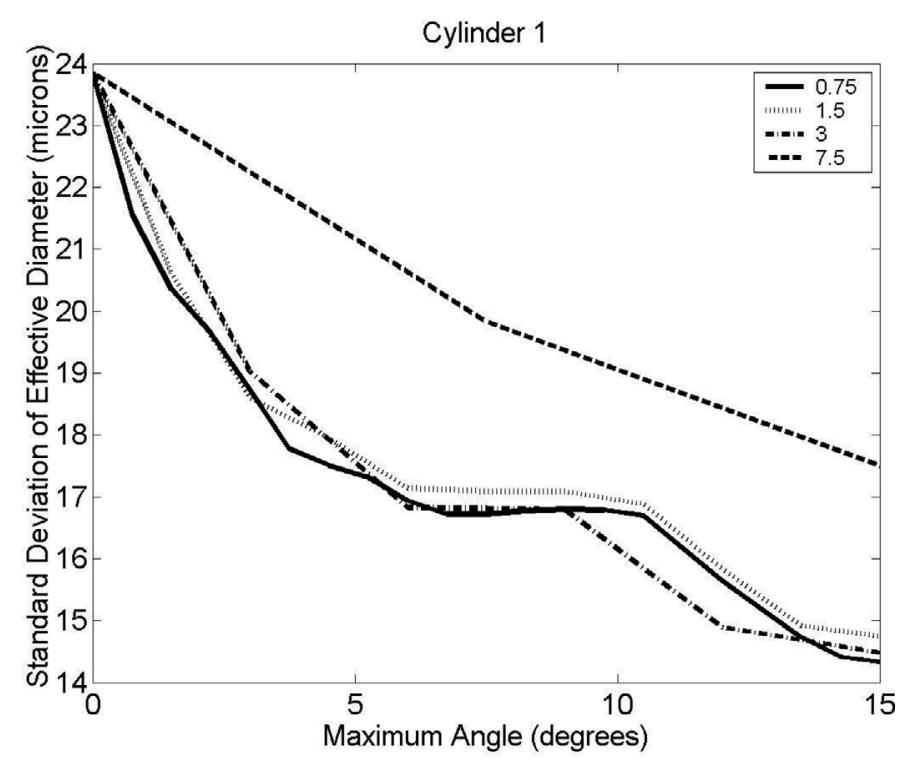

Fig. 7. Plots of compound-size estimate standard deviation for cylinder 1 as a function of the maximum angle used in compounding. Each curve corresponds to a different angular increment.

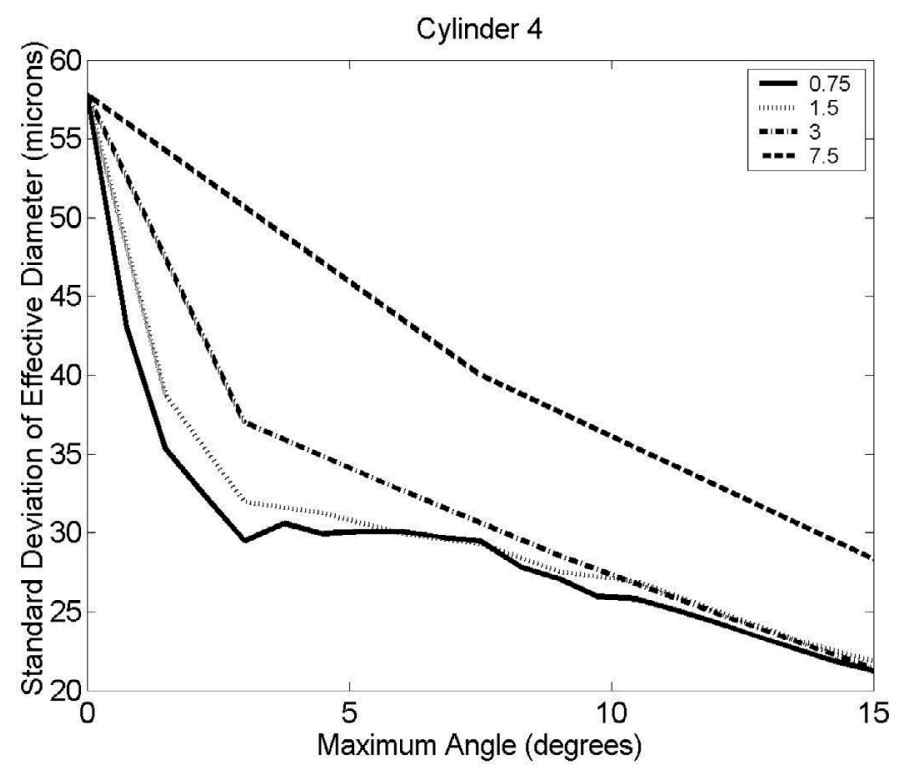

Fig. 8. Plots of compound-size estimate standard deviation for cylinder 4 as a function of the maximum angle used in compounding. Each curve corresponds to a different angular increment.

measured. The results are displayed in Fig. 10(a). Due to the method we used to compound, it is possible for the correlation to be a function of both absolute angle and angular separation. However, we found the correlation to be essentially independent of absolute angle. Fig. 10(a) seems to indicate that the correlation could be different for the four cylinders. This effect may be real and could be due to the varying depths of the cylinders. However, the measurements are too rough to make any definitive statements. In order to proceed, we ignored the effect and took the average of the four curves to be a universally applicable (i.e., independent of depth) correlation coefficient. 


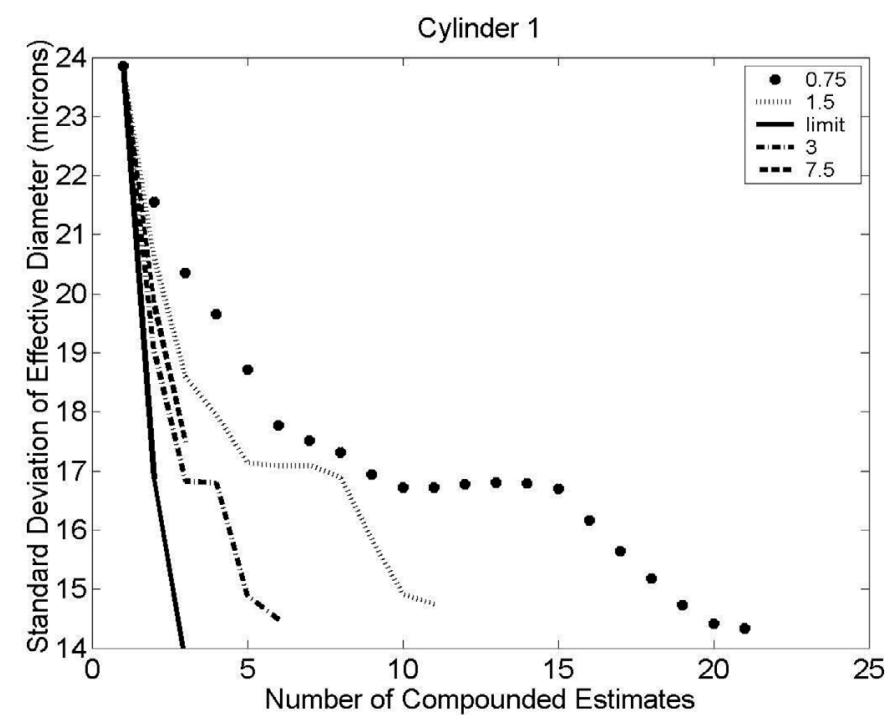

(a)

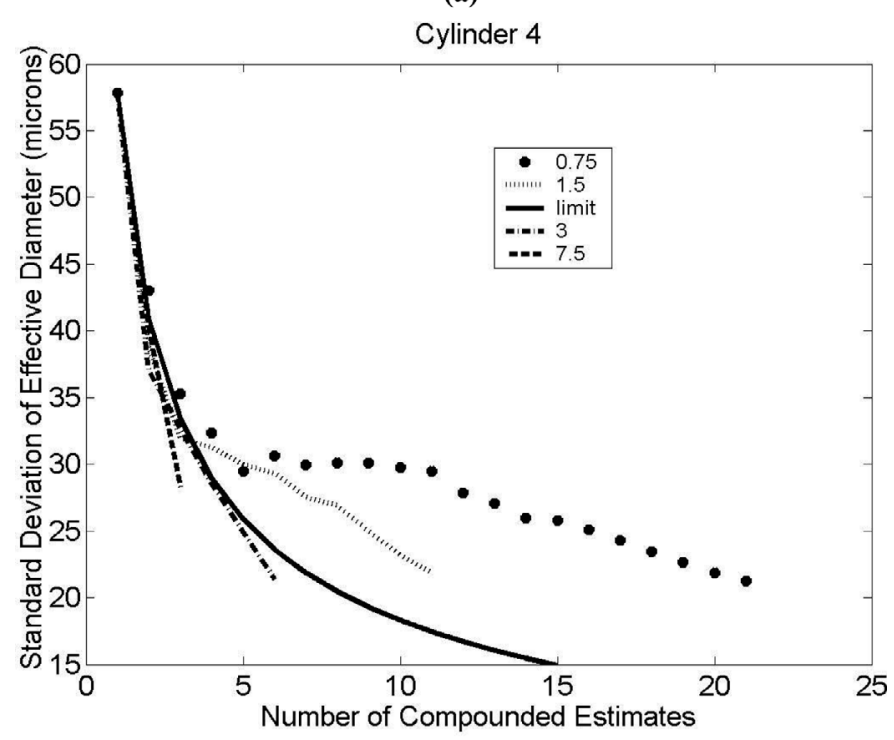

(b)

Fig. 9. Compound-size estimate standard deviation for cylinders (a) 1 and (b) 4 as a function of the number of averaged estimates for different angular increments. The displayed limiting value corresponds to the expected standard deviation when completely uncorrelated estimates are compounded.

Assuming that estimate means and variances are effectively independent of incident angle, the correlation coefficient can be used to determine the theoretical number of effective, independent, size estimates contained within a fixed maximum angle for a given angular increment according to the following [26]:

$$
N_{e f f}=\left(\frac{1}{N}+2 \sum_{i=1}^{N-1} \frac{N-i}{N^{2}} \rho_{i}\right)^{-1}
$$

where $\mathrm{N}$ is the total number of estimates contained within the maximum angle for the increment, $i$ is the angular separation between estimates in units of the increment value, and $\rho$ is the correlation coefficient. We calculated this value

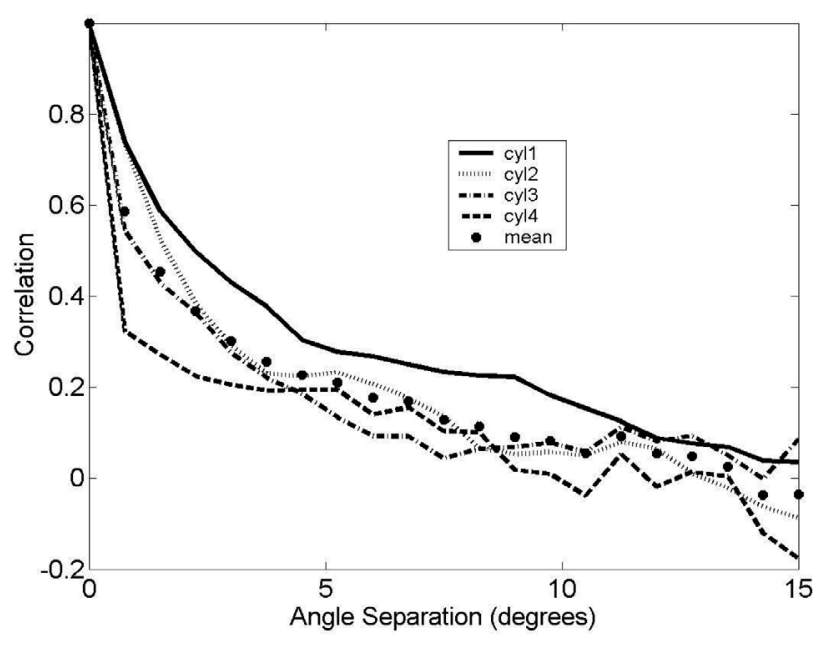

(a)

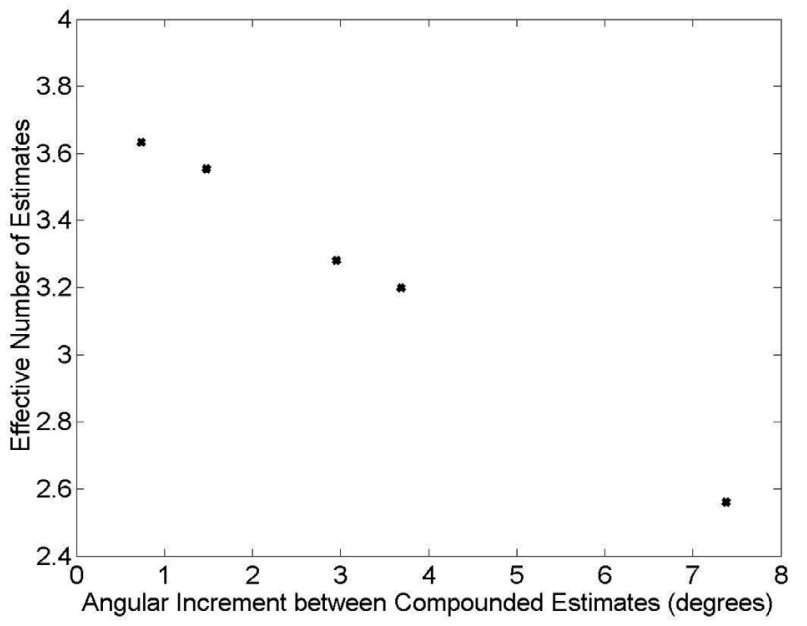

(b)

Fig. 10. Plots of (a) the correlation between size estimates as a function of angular separation for the four cylinders, and (b) the effective number of independent estimates between 0 and 15 degrees for different angular increments.

for different angular increments and a maximum angle of 15 degrees using the measured values of the correlation coefficient. The results are displayed in Fig. 10(b). Notice that the decrease in the number of effective independent estimates is steady with angular increment. Had the measured correlation coefficient dropped less drastically for smaller values of angular separation, this may not have been the case. The number of effective, independent estimates would have remained relatively constant for smaller values of angular increment, indicating that an increment value larger than the minimum could have been used for compound imaging without loss of performance (i.e., an increase in noise). However, because this was not the case in this instance, according to our calculation, performance monotonically degrades as angular increment is increased.

\section{REFERENCES}

[1] R. F. Wagner, S. W. Smith, J. M. Sandrik, and H. Lopez, "Statistics of speckle in ultrasound B-scans," IEEE Trans. Sonics Ultrason., vol. LvSU, pp. 156-163, 1983. 
[2] F. L. Lizzi, M. Ostromogilsky, E. J. Feleppa, M. C. Rorke, and M. M. Yaremko, "Relationship of ultrasonic spectral parameters to features of tissue microstructure," IEEE Trans. Ultrason., Ferroelect., Freq. Contr., vol. 34, pp. 319-329, 1987.

[3] M. F. Insana and T. J. Hall, "Characterizing the microstructure of random media using ultrasound," Phys. Med. Biol., vol. 35, pp. 1373-1386, 1990.

[4] M. F. Insana, R. F. Wagner, D. G. Brown, and T. J. Hall, "Describing small-scale structure in random media using pulse-echo ultrasound," J. Acoust. Soc. Amer., vol. 87, pp. 179-192, 1990.

[5] F. L. Lizzi, M. Astor, E. J. Feleppa, M. Shao, and A. Kalisz, "Statistical framework for ultrasonic spectral parameter imaging," Ultrasound Med. Biol., vol. 23, pp. 1371-1382, 1997.

[6] J. C. Bamber and C. R. Hill, "Acoustic properties of normal and cancerous human liver-I. Dependence on pathological condition," Ultrasound Med. Biol., vol. 7, pp. 121-133, 1981.

[7] J. C. Bamber, C. R. Hill, and J. A. King, "Acoustic properties of normal and cancerous human liver-II. Dependence of tissue structure," Ultrasound Med. Biol., vol. 7, pp. 135-144, 1981.

[8] L. L. Fellingham and F. G. Sommer, "Ultrasonic characterization of tissue structure in the in vivo human liver and spleen," IEEE Trans. Sonics Ultrason., vol. 31, pp. 418-428, 1984.

[9] Z. F. Lu, J. A. Zagzebski, and F. T. Lee, "Ultrasound backscatter and attenuation in human liver with diffuse disease," Ultrasound Med. Biol., vol. 25, pp. 1047-1054, 1999

[10] M. L. Oelze and W. D. O'Brien, Jr., "Method of improved scatterer size estimation and application to parametric imaging using ultrasound," J. Acoust. Soc. Amer., vol. 112, pp. 3053-3063, 2002.

[11] R. F. Wagner, M. F. Insana, and D. G. Brown, "Statistical properties of radio-frequency and envelope-detected signals with applications to medical ultrasound," J. Opt. Soc. Amer., vol. 4, pp. 910-922, 1987

[12] T. Varghese and K. D. Donohue, "Mean-scatterer spacing estimates with spectral correlation," J. Acoust. Soc. Amer., vol. 96, pp. 3504-3515, 1994.

[13] T. Varghese and K. D. Donohue, "Characterization of tissue microstructure scatterer distribution with spectral correlation," Ultrason. Imag., vol. 15, pp. 238-254, 1993.

[14] J. F. Chen, E. L. Madsen, and J. A. Zagzebski, "A method for determination of frequency-dependent effective scatterer number density," J. Acoust. Soc. Amer., vol. 95, pp. 77-85, 1994.

[15] J. F. Chen, J. A. Zagzebski, and E. L. Madsen, "Statistical uncertainty in estimates of an effective scatterer number density for ultrasound," J. Acoust. Soc. Amer., vol. 96, pp. 2556-2563, 1994.

16] G. E. Sleefe and P. P. Lele, "Tissue characterization based on scatterer number density estimation," IEEE Trans. Ultrason., Ferroelect., Freq. Contr., vol. 35, pp. 749-757, 1988.

[17] T. Varghese and K. D. Donohue, "Estimating mean scatterer spacing with the frequency-smoothed spectral autocorrelation function," IEEE Trans. Ultrason., Ferroelect., Freq. Contr., vol. 42, pp. 451-463, 1995

[18] M. F. Insana, T. J. Hall, J. G. Wood, and Z. Y. Yan, "Renal ultrasound using parametric imaging techniques to detect changes in microstructure and function," Invest. Radiol., vol. 28, pp. 720-725, 1993.

[19] G. Sommer, E. W. Olcott, and L. Tai, "Liver tumors: Utility of characterization at dual-frequency US," Radiology, vol. 211, pp. 629-636, 1999.

[20] M. F. Insana, T. J. Hall, and L. T. Cook, "Backscatter coefficient estimation using array transducers," IEEE Trans. Ultrason., Ferroelect., Freq. Contr., vol. 41, pp. 714-723, 1994.

[21] T. J. Hall, M. F. Insana, L. A. Harrison, and G. G. Cox, "Ultrasonic measurement of glomerular diameters in normal adult humans," Ultrasound Med. Biol., vol. 22, pp. 987-997, 1996.

[22] J. F. Chen, J. A. Zagzebski, F. Dong, and E. L. Madsen, "Estimating the spatial autocorrelation function for ultrasound scatterers in isotropic media," Med. Phys., vol. 25, pp. 648-655, 1998.

[23] A. Gerig, J. Zagzebski, and T. Varghese, "Statistics of ultrasonic scatterer size estimation with a reference phantom," J. Acoust. Soc. Amer., vol. 113, pp. 3430-3437, 2003.
[24] L. X. Yao, J. A. Zagzebski, and E. L. Madsen, "Backscatter coefficient measurements using a reference phantom to extract depth-dependent instrumentation factors," Ultrason. Imag., vol. 12 , pp. $58-70,1990$.

[25] K. K. Shung and G. A. Thieme, "Ultrasonic scattering in biological tissues," in Acoustic Scattering Theory Applied to Soft Biological Tissue. M. F. Insana and D. G. Brown, Eds. Boca Raton, FL: CRC Press, 1993, pp. 75-124.

[26] M. O'Donnell and S. D. Silverstein, "Optimum displacement for compound image generation in medical ultrasound," IEEE Trans. Ultrason., Ferroelect., Freq. Contr., vol. 35, pp. 470-476, 1988.

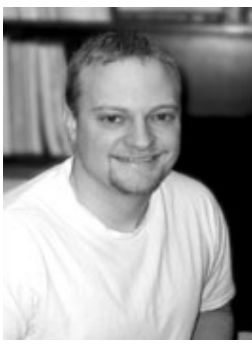

Anthony L. Gerig (S'02) was born on December 30, 1975 in Decatur, IN. He received the B.S. degree in physics and mathematics from Taylor University, Upland, IN in 1998, and the M.A. degree in physics from the University of Wisconsin-Madison in 2002. He is currently a Ph.D. candidate in physics at the U.W. Research interests include statistical signal processing, acoustic scattering and propagation, and ultrasonic beamforming and tissue characterization.

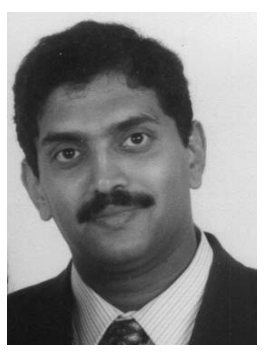

Tomy Varghese (S'92-M'95-SM'00) received the B.E. degree in instrumentation technology from the University of Mysore, Mysore, India, in 1988, and the M.S. and $\mathrm{Ph} . \mathrm{D}$. degrees in electrical engineering from the University of Kentucky, Lexington, KY, in 1992 and 1995, respectively. From 1988 to 1990 he was employed as an engineer in Wipro Information Technology Ltd., Mysore, India. From 1995 to 2000, he was a postdoctoral research associate at the Ultrasonics Laboratory, Department of Radiology, University of Texas Medical School, Houston. He is currently an assistant professor in the Department of Medical Physics and Biomedical Engineering at the University of Wisconsin-Madison.

His current research interests include elastography, ultrasound imaging, ultrasonic tissue characterization, detection and estimation theory, statistical pattern recognition, and signal and image processing applications in medical imaging. Dr. Varghese is a member of the IEEE, the American Institute of Ultrasound in Medicine (AIUM), the American Association of Physicists in Medicine (AAPM), and Eta Kappa Nu.

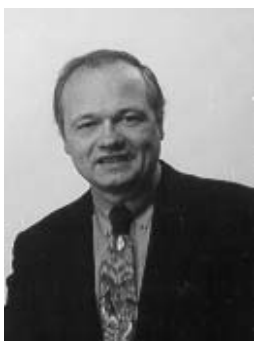

James A. Zagzebski (A'89) was born in Spevens Point, Wisconsin in 1944. He received the B.S. degree in physics from St. Mary's College, Winona, MN, and the M.S. degree in physics and the Ph.D. degree in radiological sciences from the University of Wisconsin, Madison. He is Professor of Medical Physics, Radiology, and Human Oncology at the University of Wisconsin.

His research interests include ultrasound imaging and signal processing, flow detection and visualization, and assessment of ultrasound imaging devices. Dr. Zagzebski's professional affiliations are the IEEE, the American Association of Physicists in Medicine, and the American Institute of Ultrasound in Medicine. 